\title{
From nonalcoholic fatty liver disease to metabolic dysfunction-associated fatty liver disease: more than a single-letter change in an acronym
}

\author{
Giovanni Targher \\ Department of Medicine, Section of Endocrinology, Diabetes and Metabolism, University of Verona, Verona 37126, Italy. \\ Correspondence to: Prof. Giovanni Targher, Department of Medicine, Section of Endocrinology, Diabetes and Metabolism, \\ University and Azienda Ospedaliera Universitaria Integrata, Piazzale A. Stefani 1, Verona 37126, Italy. \\ E-mail: giovanni.targher@univr.it
}

How to cite this article: Targher G. From nonalcoholic fatty liver disease to metabolic dysfunction-associated fatty liver disease: more than a single-letter change in an acronym. Hepatoma Res 2021;7:47. https://dx.doi.org/10.20517/2394-5079.2021.59

Received: 23 Apr 2021 First Decision: 13 May 2021 Revised: 13 May 2021 Accepted: 14 May 2021 Published: 11 Jun 2021

Academic Editor: Amedeo Lonardo Copy Editor: Yue-Yue Zhang Production Editor: Yue-Yue Zhang

\begin{abstract}
Nonalcoholic fatty liver disease (NAFLD) is an increasingly recognized public health problem worldwide. To emphasize the close pathophysiological links between NAFLD and overweight/obesity, insulin resistance, and related metabolic comorbidities, a consensus statement of international experts in 2020 has recommended to replace the old acronym NAFLD with "metabolic dysfunction-associated fatty liver disease" (MAFLD). A set of "positive" criteria to diagnose MAFLD, regardless of daily alcohol consumption, has also been proposed. A "positive" definition of MAFLD and its special focus on the metabolic causative drivers of this liver disease is expected to reduce patient confusion on disease etiology, which can, in turn, improve the identification and awareness of this common and burdensome liver disease among both primary care physicians and specialists. However, the proposal to change the terminology from NAFLD to MAFLD is still under intense discussion, as also recently highlighted by a panel of international experts (led by Dr. Polyzos and Mantzoros), which is the main topic of discussion of this commentary. Further studies are required to better understand whether, and how, the proposed changes to the diagnostic criteria for MAFLD may impact on the risk of adverse hepatic and extrahepatic clinical outcomes.
\end{abstract}

Keywords: Nonalcoholic fatty liver disease, metabolic dysfunction-associated fatty liver disease 
Nonalcoholic fatty liver disease (NAFLD) has reached epidemic proportions and is the commonest cause of chronic liver diseases in Western countries ${ }^{[1-3]}$. Based on epidemiological and pathophysiological insights gained from the past decades, an international panel of experts in 2020 has proposed a change of terminology and definition for NAFLD, called "metabolic dysfunction-associated fatty liver disease" $(\mathrm{MAFLD})^{[4,5]}$.

During the last 40 years, there have been concerns expressed by several individual experts and some special conferences of the American Association for the Study of Liver Diseases and European Association for the Study of the Liver societies regarding the inaccuracy and possible "negative" implications of using the term "NAFLD" to describe a fatty liver disease that is often associated with overweight/obesity, insulin resistance, and related metabolic disorders, including type 2 diabetes mellitus (T2DM), as extensively discussed in ${ }^{[6,7]}$. In particular, it has been repeatedly pointed out that the diagnosis of NAFLD is always a diagnosis of exclusion (it only exists when all other competing causes for hepatic steatosis are excluded), and the term NAFLD does not capture the high heterogeneity of this patient population with regard to its main metabolic drivers and disease modifiers (i.e., overweight/obesity, metabolic syndrome, insulin resistance, and $\mathrm{T} 2 \mathrm{DM})^{[4,5,8-10]}$. Furthermore, the term NAFLD does not define an individual phenotype precisely for the development of targeted pharmacologic therapies (with variable impacts on randomized clinical trials and suboptimal/poor responses to treatments and trial failure). In addition, the adjective "nonalcoholic" often trivializes and/or discriminates the persons affected by this liver disease ${ }^{[4,5,8-10]}$. As regards to this, previous studies have shown that up to nearly $60 \%-65 \%$ of adults in the United States and in other Western countries have a moderate consumption of alcoholic beverages, consuming over four drinks a week ${ }^{[1,12]}$.

For NAFLD, it is currently assumed that the daily amount of alcohol consumed is below the thresholds (i.e., conventionally defined as $<30 \mathrm{~g} /$ day for men and $<20 \mathrm{~g} /$ day for women $)^{[13]}$, where alcohol consumption would have a significant impact on clinical phenotype, disease progression, and outcomes. However, growing epidemiological evidence clearly suggests that the safe limit of alcohol consumption is zero ${ }^{[12,14]}$. Thus, it is evident that, in routine clinical practice, NAFLD does often coexist with a mild-to-moderate alcohol consumption that may also exert synergistic or additive adverse effects on clinical outcomes of this patient population ${ }^{[15,16]}$. Finally, there is currently a lack of patient awareness, public health response, and underfunding for $\mathrm{NAFLD}^{[17,18]}$.

That said, polarizing opinions and major debate have recently arisen in hepatology on the proposed change of nomenclature and diagnostic criteria for MAFLD ${ }^{[8-10,19-21]}$. In this context, a recent commentary written by an international panel of experts, led by Polyzos et al..$^{[22]}$, has recognized that the newly proposed definition of MAFLD is expected to more accurately reflect the heterogeneous pathogenesis of this liver disease, focusing on the metabolic abnormalities which play a key role in its development and progression. The authors of this commentary also recognize the clinical relevance to propose a diagnosis of disease that is expressed in "positive" (i.e., metabolic dysfunction-associated fatty liver) and not "negative" terms signifying exclusion (nonalcoholic fatty liver without coexisting known causes of fatty liver) ${ }^{[2]}$. However, despite the need for a change in terminology and definition of this metabolic liver disease, these authors suggested to proceed cautiously in order to minimize any potential adverse implications for a premature change in terminology of NAFLD. In addition, they also stressed that there is still significant ambiguity in the definition of MAFLD ${ }^{[22]}$.

The new definition of MAFLD is based on "positive" diagnostic criteria, according to which all Caucasian adults with hepatic steatosis (as detected by liver histology, imaging methods, or blood biomarkers/scores) and body mass index (BMI) $\geq 25 \mathrm{~kg} / \mathrm{m}^{2}$ (or $\geq 23 \mathrm{~kg} / \mathrm{m}^{2}$ in Asian people) and/or established T2DM have 
MAFLD, whereas lean and nondiabetic individuals with hepatic steatosis should fulfill at least two of seven metabolic risk abnormalities (that are widely used to identify the metabolic syndrome, using country- and ethnic-specific cutoff points of increased waist circumference) to be diagnosed as MAFLD ${ }^{[5]}$. One of the advantages of this definition is that it does not rely on exclusion of significant alcohol consumption and other competing causes of hepatic steatosis to establish the diagnosis ${ }^{[5]}$. Persons with MAFLD with one (or more) coexisting causes of hepatic steatosis should be defined as having dual (or more) etiology of fatty liver disease $\mathrm{e}^{[5]}$. In the new definition of MAFLD, a set of diagnostic criteria to define MAFLD-related cirrhosis has also been proposed (to avoid the use of the old term of "cryptogenic cirrhosis" in this group of patients), as well as a conceptual framework to consider other causes of fatty liver disease ${ }^{[5]}$.

Among the possible ambiguities in the newly proposed definition of MAFLD, Polyzos et al. ${ }^{[22]}$ of the aforementioned commentary firstly suggested that the use of both BMI and waist circumference adds an unnecessary layer, complicating the definition of MAFLD. Then, they proposed either waist circumference or direct assessment of percent fat mass via dual energy X-absorptiometry (DEXA) should replace BMI in the definition of overweight/obesity ${ }^{[2]}$. Secondly, given that the exclusion of patients with concomitant liver diseases (e.g., alcohol, virus, and drugs) is not a prerequisite for the diagnosis of MAFLD (which is a key differentiation as compared with the NAFLD definition), they proposed to exclude these subgroups of patients with mixed causes of hepatic steatosis that are still under-studied, in order to further reduce the heterogeneity of MAFLD ${ }^{[2]}$. Thirdly, they suggested that the cut-offs proposed for the diagnosis of the seven metabolic risk abnormalities of MAFLD among lean and nondiabetic individuals (that are widely used for the definition of the metabolic syndrome) might create some degree of bias in the definition of MAFLD, until specific cut-off points for MAFLD are validated ${ }^{[22]}$. A final issue raised by the authors of this commentary is about the proposed diagnosis of the histological severity of MAFLD that should be based exclusively on the grade of activity and the stage of liver fibrosis, thus resulting in the abolishment of the term nonalcoholic steatohepatitis (NASH) ${ }^{[2]}$. In fact, the new definition of MAFLD recognizes that the grade of MAFLD activity is a disease continuum, and the all-embracing term of MAFLD should replace the stratification of the liver disease into NASH and non-NASH, which may have some important diagnostic limitations, possibly due to substantial intra- and inter-observer variabilities for the histologic diagnosis of $\mathrm{NASH}^{[5]}$. As regards this issue, the authors of the commentary suggested that the abolishment of NASH might create confusion in terms of the ongoing randomized clinical trials testing the efficacy of novel pharmacological therapies in individuals with NASH, but might also make results of published clinical trials impossible to interpret ${ }^{[22]}$.

Personally, I agree with some but not all of the issues raised by the authors of this elegant commentary. For example, I disagree with their proposal that either waist circumference or direct assessment of percent fat mass via DEXA should replace BMI in the definition of overweight/obesity. This would markedly reduce the broad applicability of the MAFLD definition in routine clinical practice. Moreover, I also partly disagree that the cut-offs proposed for the diagnosis of the seven metabolic risk abnormalities of MAFLD among lean and nondiabetic individuals might create a degree of bias in the definition of MAFLD. That said, it is reasonable to assume that diagnosis of MAFLD should be sub-phenotyped in the near future, mostly based on the predominant pathophysiological pathways driving the development of individual liver histology features (hepatic steatosis, inflammation, or fibrosis), which might differentially affect the risk of liverrelated complications and some extra-hepatic clinical outcomes, such as cardiovascular disease, chronic kidney disease, or extra-hepatic malignancies.

It is important to further underline that the proposed change of nomenclature from NAFLD to MAFLD is more than a simple change of a single letter in an acronym ${ }^{[7,23]}$. Although there is an excellent concordance 
between the MAFLD and NAFLD definitions, it has recently been demonstrated that these two definitions are not fully interchangeable ${ }^{[24-27]}$. In fact, comparing the MAFLD and NAFLD diagnostic criteria by using "real-world" databases, there are at least two new categories of individuals with fatty liver disease: (1) subjects with MAFLD and other coexisting chronic liver diseases who do not have NAFLD; and (2) lean individuals with NAFLD, who do not have MAFLD and coexisting metabolic dysfunction ${ }^{[24]}$. These new categories of individuals will need to be carefully studied in the near future to assess the natural history of their liver disease, the role of genetic predisposition, and the risk of developing hepatic and extra-hepatic complications over time. This will likely also allow new discoveries of causes, mechanisms, and treatments of fatty liver disease. Notably, emerging studies comparing the diagnostic performance of the NAFLD and MAFLD definitions also suggest that, compared with the NAFLD definition, MAFLD may better identify individuals with hepatic steatosis at higher risk of disease progression (i.e., those with significant/advanced liver fibrosis $)^{[25,28]}$ or individuals who have a greater risk of extra-hepatic complications, such as cardiovascular disease or chronic kidney disease ${ }^{[29-31]}$. Other studies also showed that MAFLD is superior to NAFLD in identifying patients with fibrosis among those with co-existing other liver diseases, such as chronic viral hepatitis $\mathrm{B}$ and $\mathrm{C}$ or persons with human immunodeficiency virus ${ }^{[32,33]}$. In addition, an international panel of experts have recently highlighted the importance to taking into consideration the overlooked impact of MAFLD for reducing morbidity and mortality in people who have had chronic viral hepatitis $\mathrm{C}^{[34]}$.

Collectively, it is important to further underline that the proposed change of terminology from NAFLD to MAFLD is not simply a semantic revision (or a single-letter change in an acronym), but it may facilitate improved diagnosis of this common and burdensome liver disease for better health promotion and case identification, as well as decreased social stigmatization, improved health services delivery, and allocated funding ${ }^{[5,8,9,23,3,3]}$. The change from NAFLD to MAFLD may also increase the awareness of fatty liver disease in both primary care providers and other specialists ${ }^{[36,37]}$. Furthermore, it is reasonable to hypothesize that the use of the new definition for MAFLD and the exclusion of individuals with hepatic steatosis without coexisting metabolic dysfunction will render study cohorts more homogeneous, thereby further improving the response rates of pharmacological treatments for this common metabolic liver disease ${ }^{[5,8,9,35]}$.

As elegantly espoused by Eslam and George ${ }^{[38]}$, among the many positive attributes of the proposed change of terminology from NAFLD to MAFLD, we should not lose sight of the fact that it has generated a momentum of its own that should now be encouraged rather than emasculated. The debates are inevitable and also very useful in science, but they should be mostly based on the evidence of data rather than the eminence of opinions, because only the evidence of data can settle them and clear the way forward ${ }^{[19-23,39-41]}$ In the era of evidence-based medicine, the need for an evidence-based debate on the proposed change of NAFLD terminology is clinically essential ${ }^{[21]}$. Very recently, some scientific societies have reached a consensus to endorse the proposal that NAFLD should be renamed as MAFLD and that this metabolic liver disease should be diagnosed by simple and easily applicable "positive" criteria ${ }^{[42-45]}$. Furthermore, the new definition of MAFLD has received endorsement from international patient advocacy groups as well as from a nurse and allied health perspective ${ }^{[46,47]}$. Some authors have also endorsed the new terminology of MAFLD for the use in the pediatric population and proposed a set of specific cut-off points, which might be useful to diagnose MAFLD in children and adolescents ${ }^{[48]}$. However, I believe that it is mandatory that an international consensus conference should be jointly organized by the "major" relevant scientific societies of hepatology/gastroenterology, diabetology, and obesity along with the patient advocacy organizations, biopharmaceutical industry, regulatory agencies, and policy makers to deeply discuss all potential implications of a change of terminology from NAFLD to MAFLD ${ }^{[5,7,19]}$. 


\section{DECLARATIONS}

\section{Authors' contributions}

The author contributed solely to the article.

\section{Availability of data and materials}

Not applicable.

\section{Financial support and sponsorship}

None.

\section{Conflicts of interest}

The author declared that there are no conflicts of interest. Targher $G$ was a coauthor of the recent article ${ }^{[5]}$ proposing the new definition for MAFLD.

\section{Ethical approval and consent to participate}

Not applicable.

\section{Consent for publication}

Not applicable.

\section{Copyright}

(c) The Author(s) 2021.

\section{REFERENCES}

1. Younossi Z, Anstee QM, Marietti M, et al. Global burden of NAFLD and NASH: trends, predictions, risk factors and prevention. Nat Rev Gastroenterol Hepatol 2018;15:11-20. DOI PubMed

2. Araújo AR, Rosso N, Bedogni G, Tiribelli C, Bellentani S. Global epidemiology of non-alcoholic fatty liver disease/non-alcoholic steatohepatitis: What we need in the future. Liver Int 2018;38 Suppl 1:47-51. DOI PubMed

3. Mantovani A, Scorletti E, Mosca A, Alisi A, Byrne CD, Targher G. Complications, morbidity and mortality of nonalcoholic fatty liver disease. Metabolism 2020;111S:154170. DOI PubMed

4. Eslam M, Sanyal AJ, George J; International Consensus Panel. MAFLD: A consensus-driven proposed nomenclature for metabolic associated fatty liver disease. Gastroenterology 2020;158:1999-2014.e1. DOI PubMed

5. Eslam M, Newsome PN, Sarin SK, et al. A new definition for metabolic dysfunction-associated fatty liver disease: an international expert consensus statement. J Hepatol 2020;73:202-9. DOI PubMed

6. Lonardo A, Leoni S, Alswat KA, Fouad Y. History of Nonalcoholic Fatty Liver Disease. Int J Mol Sci 2020;21:5888. DOI PubMed PMC

7. Targher G, Byrne CD. From nonalcoholic fatty liver disease to metabolic dysfunction-associated fatty liver disease: is it time for a change of terminology? Hepatoma Res 2020;6:64. DOI

8. Fouad Y, Waked I, Bollipo S, Gomaa A, Ajlouni Y, Attia D. What's in a name? Liver Int 2020;40:1254-61. DOI PubMed

9. Valenti L, Pelusi S. Redefining fatty liver disease classification in 2020. Liver Int 2020;40:1016-7. DOI PubMed

10. Tilg H, Effenberger M. From NAFLD to MAFLD: when pathophysiology succeeds. Nat Rev Gastroenterol Hepatol 2020;17:387-8. DOI PubMed

11. Dawson DA, Goldstein RB, Saha TD, Grant BF. Changes in alcohol consumption: United States, 2001-2002 to 2012-2013. Drug Alcohol Depend 2015;148:56-61. DOI PubMed PMC

12. Griswold MG, Fullman N, Hawley C, et al. Alcohol use and burden for 195 countries and territories, 1990-2016: a systematic analysis for the Global Burden of Disease Study 2016. Lancet 2018;392:1015-35. DOI PubMed PMC

13. Association for the Study of the Liver (EASL); European Association for the Study of Diabetes (EASD); European Association for the Study of Obesity (EASO). EASL-EASD-EASO Clinical Practice Guidelines for the management of non-alcoholic fatty liver disease. $J$ Hepatol 2016;64:1388-402. DOI PubMed

14. Chang Y, Cho YK, Kim Y, et al. Nonheavy drinking and worsening of noninvasive fibrosis markers in nonalcoholic fatty liver disease: a COHORT study. Hepatology 2019;69:64-75. DOI PubMed

15. Khoudari G, Singh A, Noureddin M, et al. Characterization of patients with both alcoholic and nonalcoholic fatty liver disease in a large United States cohort. World J Hepatol 2019;11:710-8. DOI PubMed PMC

16. Åberg F, Helenius-Hietala J, Puukka P, Färkkilä M, Jula A. Interaction between alcohol consumption and metabolic syndrome in predicting severe liver disease in the general population. Hepatology 2018;67:2141-9. DOI PubMed

17. Wieland AC, Mettler P, McDermott MT, Crane LA, Cicutto LC, Bambha KM. Low awareness of nonalcoholic fatty liver disease 
among patients at high metabolic risk. J Clin Gastroenterol 2015;49:e6-e10. DOI PubMed

18. Lazarus JV, Ekstedt M, Marchesini G, et al; EASL International Liver Foundation NAFLD Policy Review Collaborators. A crosssectional study of the public health response to non-alcoholic fatty liver disease in Europe. J Hepatol 2020;72:14-24. DOI PubMed

19. Younossi ZM, Rinella ME, Sanyal AJ, et al. From NAFLD to MAFLD: Implications of a Premature Change in Terminology. Hepatology 2021;73:1194-8. DOI PubMed

20. Lonardo A. Renaming NAFLD to MAFLD: Could the LDE system assist in this transition? J Clin Med 2021;10:492. DOI PubMed PMC

21. Fouad Y, Elwakil R, Elsahhar M, et al. The NAFLD $\square$ MAFLD debate: Eminence vs evidence. Liver Int 2021;41:255-60. DOI PubMed

22. Polyzos SA, Kang ES, Tsochatzis EA, et al. Commentary: Nonalcoholic or metabolic dysfunction-associated fatty liver disease? Metabolism 2020;113:154413. DOI PubMed

23. Yilmaz Y, Byrne CD, Musso G. A single-letter change in an acronym: signals, reasons, promises, challenges, and steps ahead for moving from NAFLD to MAFLD. Expert Rev Gastroenterol Hepatol 2021;15:345-52. DOI PubMed

24. Targher G. Concordance between MAFLD and NAFLD diagnostic criteria in 'real-world' data. Liver Int 2020;40:2879-80. DOI PubMed

25. Lin S, Huang J, Wang M, et al. Comparison of MAFLD and NAFLD diagnostic criteria in real world. Liver Int 2020;40:2082-9. DOI PubMed

26. Ciardullo S, Perseghin G. Prevalence of NAFLD, MAFLD and associated advanced fibrosis in the contemporary United States population. Liver Int 2021;41:1290-3. DOI PubMed

27. Wai-Sun Wong V, Lai-Hung Wong G, Woo J, et al. Impact of the New Definition of Metabolic Associated Fatty Liver Disease on the Epidemiology of the Disease. Clin Gastroenterol Hepatol 2020:S1542-3565(20)31504. DOI PubMed

28. Yamamura S, Eslam M, Kawaguchi T, et al. MAFLD identifies patients with significant hepatic fibrosis better than NAFLD. Liver Int 2020;40:3018-30. DOI PubMed

29. Lee H, Lee YH, Kim SU, Kim HC. Metabolic Dysfunction-Associated Fatty Liver Disease and Incident Cardiovascular Disease Risk: A Nationwide Cohort Study. Clin Gastroenterol Hepatol 2020:S1542-3565(20)31717. DOI PubMed

30. Niriella MA, Ediriweera DS, Kasturiratne A, et al. Outcomes of NAFLD and MAFLD: Results from a community-based, prospective cohort study. PLoS One 2021;16:e245762. DOI PubMed PMC

31. Sun DQ, Jin Y, Wang TY, et al. MAFLD and risk of CKD. Metabolism 2021;115:154433. DOI PubMed

32. Mak LY, Yuen MF, Seto WK. Letter regarding "A new definition for metabolic dysfunction-associated fatty liver disease: An international expert consensus statement". J Hepatol 2020;73:1573-4. DOI PubMed

33. Liu D, Shen Y, Zhang R, et al. Prevalence and risk factors of metabolic associated fatty liver disease among people living with HIV in China. J Gastroenterol Hepatol 2020. DOI PubMed

34. Fouad Y, Lazarus JV, Negro F, et al. MAFLD considerations as a part of the global hepatitis C elimination effort: an international perspective. Aliment Pharmacol Ther 2021;53:1080-9. DOI PubMed

35. Zheng KI, Fan JG, Shi JP, et al. From NAFLD to MAFLD: a "redefining" moment for fatty liver disease. Chin Med J (Engl) 2020;133:2271-3. DOI PubMed PMC

36. Fouad Y, Gomaa A, Semida N, Ghany WA, Attia D. Change from NAFLD to MAFLD increases the awareness of fatty liver disease in primary care physicians and specialists. J Hepatol 2021;74:1254-6. DOI PubMed

37. Méndez-Sánchez N, Díaz-Orozco L, Córdova-Gallardo J. Redefinition of fatty liver disease from NAFLD to MAFLD raised disease awareness: Mexican experience. J Hepatol 2021:S0168-8278(21)00256. DOI PubMed

38. Eslam M, George J. MAFLD: Now is the time to capitalize on the momentum. J Hepatol 2021;74:1262-3. DOI PubMed

39. Singh SP, Anirvan P, Reddy KR, et al. Non-alcoholic fatty liver disease: Not time for an obituary just yet! J Hepatol 2021;74:972-4. DOI PubMed

40. Kang SH, Cho Y, Jeong SW, Kim SU, Lee JW; Korean NAFLD Study Group. From nonalcoholic fatty liver disease to metabolicassociated fatty liver disease: Big wave or ripple? Clin Mol Hepatol 2021;27:257-69. DOI PubMed PMC

41. Yilmaz Y. Metabolic-associated fatty liver disease: Time to integrate ground-breaking new terminology to our clinical practice? Hepatology Forum 2020;3:79-81. DOI

42. Eslam M, Sarin SK, Wong VW, et al. The Asian Pacific Association for the Study of the Liver clinical practice guidelines for the diagnosis and management of metabolic associated fatty liver disease. Hepatol Int 2020;14:889-919. DOI PubMed

43. Shiha G, Alswat K, Al Khatry M, et al. Nomenclature and definition of metabolic-associated fatty liver disease: a consensus from the Middle East and north Africa. Lancet Gastroenterol Hepatol 2021;6:57-64. DOI PubMed

44. Mendez-sanchez N, Arrese M, Gadano A, et al. The Latin American Association for the Study of the Liver (ALEH) position statement on the redefinition of fatty liver disease. Lancet Gastroenterol Hepatol 2021;6:65-72. DOI PubMed

45. Spearman CW, Desalegn H, Ocama P, et al. The sub-Saharan Africa position statement on the redefinition of fatty liver disease: From NAFLD to MAFLD. J Hepatol 2021;74:1256-8. DOI PubMed

46. Shiha G, Korenjak M, Eskridge W, et al. Redefining fatty liver disease: an international patient perspective. Lancet Gastroenterol Hepatol 2021;6:73-9. DOI PubMed

47. Clayton M, Fabrellas N, Luo J, et al. From NAFLD to MAFLD: Nurse and allied health perspective. Liver Int 2021;41:683-91. DOI PubMed

48. Flisiak-Jackiewicz M, Bobrus-Chociej A, Wasilewska N, Lebensztejn DM. From nonalcoholic fatty liver disease (NAFLD) to metabolic dysfunction-associated fatty liver disease (MAFLD)-new terminology in pediatric patients as a step in good scientific 

direction? J Clin Med 2021;10:924. DOI PubMed PMC 